\title{
Interwell relaxation times in $p$-Si/SiGe asymmetric quantum well structures: Role of interface roughness
}

\author{
Marco Califano, ${ }^{1}$ N. Q. Vinh, ${ }^{2}$ P. J. Phillips,${ }^{3}$ Z. Ikonić, ${ }^{1}$ R. W. Kelsall, ${ }^{1}$ P. Harrison, ${ }^{1}$ C. R. Pidgeon, ${ }^{3}$ B. N. Murdin, ${ }^{4}$ \\ D. J. Paul, ${ }^{5}$ P. Townsend, ${ }^{5}$ J. Zhang, ${ }^{6}$ I. M. Ross, ${ }^{7}$ and A. G. Cullis ${ }^{7}$ \\ ${ }^{1}$ Institute of Microwaves and Photonics, School of Electronic and Electrical Engineering, University of Leeds, Leeds, LS2 9JT, \\ United Kingdom \\ ${ }^{2}$ FOM Institute for Plasma Physics “Rijnhuizen," P. O. Box 1207, NL-3430 BE Nieuwegein, The Netherlands \\ ${ }^{3}$ Department of Physics, Heriot-Watt University, Edinburgh, EH14 4AS, United Kingdom \\ ${ }^{4}$ Advanced Technology Institute, University of Surrey, Guildford, GU2 7XH, United Kingdom \\ ${ }^{5}$ Cavendish Laboratory, University of Cambridge, Cambridge, CB3 OHE, United Kingdom \\ ${ }^{6}$ Department of Physics, Imperial College, London, SW7 2BZ, United Kingdom \\ ${ }^{7}$ Department of Electronic and Electrical Engineering, University of Sheffield, Sheffield, S1 3JD, United Kingdom
}

(Received 29 September 2006; published 24 January 2007)

\begin{abstract}
We report the direct determination of nonradiative lifetimes in $\mathrm{Si} / \mathrm{SiGe}$ asymmetric quantum well structures designed to access spatially indirect (diagonal) interwell transitions between heavy-hole ground states, at photon energies below the optical phonon energy. We show both experimentally and theoretically, using a six-band $\mathbf{k} \cdot \mathbf{p}$ model and a time-domain rate equation scheme, that, for the interface quality currently achievable experimentally (with an average step height $\geqslant 1 \AA$ ), interface roughness will dominate all other scattering processes up to about $200 \mathrm{~K}$. By comparing our results obtained for two different structures we deduce that in this regime both barrier and well widths play an important role in the determination of the carrier lifetime. Comparison with recently published experimental and theoretical data obtained for mid-infrared $\mathrm{GaAs} / \mathrm{Al}_{x} \mathrm{Ga}_{1-x} \mathrm{As}$ multiple quantum well systems leads us to the conclusion that the dominant role of interface roughness scattering at low temperature is a general feature of a wide range of semiconductor heterostructures not limited to IV-IV materials.
\end{abstract}

DOI: 10.1103/PhysRevB.75.045338

PACS number(s): 78.47.+p, 71.20.-b

\section{INTRODUCTION}

Following the success of III-V quantum cascade lasers (QCLs) at both mid-infrared ${ }^{1}$ and far-infrared (terahertz) frequencies, ${ }^{2,3}$ there is considerable interest in the development of silicon-based quantum cascade devices for low-cost sources and optoelectronic integration with other circuitry. The indirect band gap that has hindered interband emitter development in $\mathrm{Si}, \mathrm{Si}_{1-x} \mathrm{Ge}_{x}$, and $\mathrm{Ge}$ does not affect QCLs, as they rely on carrier transitions within the same (conduction or valence) band rather than on exciton recombination across the band gap. Indeed, SiGe-based materials have a major advantage over III-V's for this type of device, in that phonons are nonpolar, ${ }^{4}$ and this reduces the principal nonradiative scattering rate that limits the efficiency in III-V devices. ${ }^{2}$ This is particularly true at photon energies below the optical phonon energy $\left(\hbar \omega<\hbar \omega_{o p t}\right)$ which range is the subject of the present work.

If a $\mathrm{QCL}$ could be realized in $\mathrm{Si} / \mathrm{SiGe}$, the lack of polar optical phonon scattering, the lower free carrier absorption, and the higher thermal conductivity of Si could allow significantly higher operating temperatures in the terahertz $(\mathrm{THz})$ range. Moreover, there could be lower manufacturing costs involved with the mature silicon process technology. We have previously demonstrated the first electroluminescence from $p$-Si/SiGe quantum cascade emitters at $\mathrm{THz}$ frequencies, ${ }^{5}$ however, no significant optical gain or stimulated emission has been achieved so far in $\mathrm{Si} / \mathrm{SiGe} \mathrm{QC}$ structures. In order to achieve a silicon-based QCL, a structure that can achieve population inversion and high gain has to be designed. Of paramount importance to this end is the ability to engineer carrier lifetimes.

The intersubband lifetimes of both inter- and intrawell transitions can be tailored by carefully designing the thickness and the composition of the different layers that form the QC structure. A tunability with barrier width has been demonstrated previously at energies above $\hbar \omega_{\text {opt }}$, where lifetimes varying between 20 and 35 ps were deduced from electroluminescence measurements for diagonal transitions in QC structures of $p$-Si/SiGe. ${ }^{6}$ However, the transport properties in semiconductor quantum wells have also been shown ${ }^{7-12}$ to be strongly influenced by the quality of the interfaces. Fluctuations in the well width due to the presence of nonideal surfaces result in local fluctuations of the carriers' confinement potential, which act as a scattering potential for the two-dimensional (2D) carrier gas. A very recent detailed experimental and theoretical study on $n$-GaAs $/ \mathrm{Al}_{x} \mathrm{Ga}_{1-x} \mathrm{As}$ mid-infrared QCLs found ${ }^{13}$ that interface roughness (IFR) scattering is the most efficient elastic relaxation mechanism at low temperature in those systems and becomes dominant whenever LO-phonon emission is inhibited. Its inclusion in the theoretical calculations was shown to be crucial to reproduce the observed magnetic field dependence of the emitted power. In $\mathrm{SiGe}$ systems, previous theoretical ${ }^{9}$ and experimental ${ }^{11}$ studies have found that IFR scattering is a major limiting factor for the low-temperature mobility of 2D hole gases in quantum wells (QWs) narrower than about $5 \mathrm{~nm}$.

Here we present the results of a theoretical and experimental investigation into the effects of IFR scattering on the 
lifetime of diagonal (i.e., interwell) intersubband transitions between heavy-hole (HH) ground states in two different $p$ -SiGe coupled asymmetric quantum wells (CAQWs) in this size range. The experimental information is provided by time-resolved measurements with a far-infrared pulsed freeelectron laser. Such a device is the only laser source that has a short enough pulse duration and high enough intensity to pump and probe the fast recovery transition in this wavelength range. It also provides wide wavelength coverage for $\mathrm{HH}-\mathrm{HH}$ and $\mathrm{HH}-$ light hole (LH) transitions, and continuous tunability for resonant excitation. ${ }^{4}$ In contrast, the previously reported data on decay times in $p$-Si/SiGe QC structures ${ }^{6}$ were obtained indirectly from electroluminescence measurements. The extraction of subband lifetimes in that case was therefore based on a number of assumptions which may have limited the accuracy of the deduced values. Here, by using a pump-probe technique, we measure directly the decay time from the upper $\mathrm{HH} 1$ ground state to the lower $\mathrm{HH} 1$ ground state in the adjacent quantum well. Furthermore, unlike in Ref. 6, in the present case the structures are specifically designed so that between these two levels there is no other state that could provide alternative relaxation pathways, allowing an unambiguous determination of the upper-state lifetime. We show that we can achieve very long lifetimes $(\sim 70 \mathrm{ps})$, which exhibit a relative insensitivity to the temperature compared to GaAs devices. ${ }^{2}$

We calculate IFR scattering rates, compare them with the rates of other mechanisms, such as scattering due to alloy disorder and acoustic and optical phonons as well as carriercarrier interaction (all calculated within a time-domain rate equation scheme ${ }^{4}$ ) and predict that, despite the good quality of our interfaces (with an average roughness step height of only $1.2-1.5 \AA$ ), IFR will constitute more than $50 \%$ of the total scattering rate up to $\sim 100 \mathrm{~K}$, remaining stronger than any of the other scattering processes up to about $200 \mathrm{~K}$. We show that in this regime both barrier and well widths need to be carefully designed in order to achieve the desired intersubband transition rates. Our theoretical results are in very good agreement with our measured lifetimes in the temperature range experimentally investigated.

\section{EXPERIMENTAL DETAILS}

We have investigated two strain-symmetrized $\mathrm{Si} / \mathrm{SiGe}$ CAQW structures on (001) $\mathrm{Si}$, utilizing pump-probe spectroscopy of $\mathrm{HH}$ interwell transitions to measure the nonradiative recombination by diagonal (bound to bound) HH1-HH1 transitions through the $\mathrm{Si}$ barrier. The structures considered in the present study (BF1499 and BF1500) consist of 20 periods each containing CAQWs grown on a relaxed buffer of $\mathrm{Si}_{0.8} \mathrm{Ge}_{0.2}$. The first (BF1499) has 3.4 and $4.6 \mathrm{~nm}$ undoped $\mathrm{SiGe}$ wells with a nominal Ge content of $40 \%$, separated by a Si barrier of $1.8 \mathrm{~nm}$. The second wafer (BF1500) has 3.2 and $4.15 \mathrm{~nm}$ undoped $\mathrm{SiGe}$ wells with a nominal Ge content of $40 \%$, separated by a $2.3 \mathrm{~nm} \mathrm{Si}$ barrier. The pairs of CAQWs are separated by a $5 \mathrm{~nm} \mathrm{Si}$ barrier, the central $3.2 \mathrm{~nm}$ of which is modulation doped with $\mathrm{B}$ to a concentration of $N_{A}=1.6 \times 10^{18} \mathrm{~cm}^{-3}$. The layer thicknesses have been measured by transmission electron microscopy (TEM) using

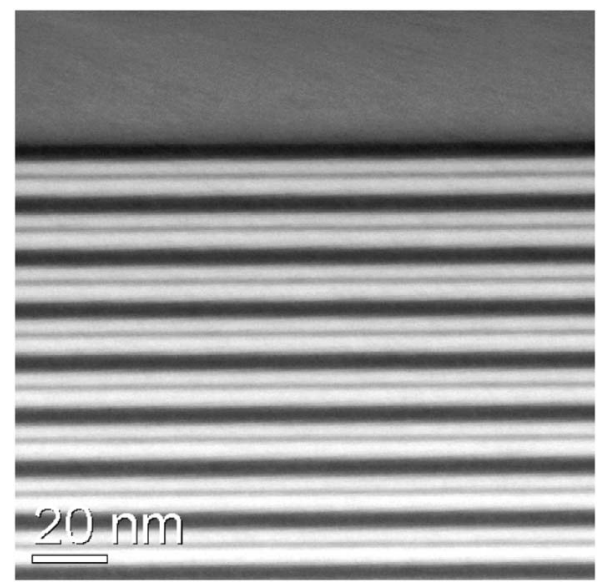

FIG. 1. Annular dark field scanning transmission electron micrograph showing the first eight sets of pairs of QWs and the barriers for the first structure (BF1499).

high-angle angular dark field (HAADF) imaging (see Fig. 1), while the as-grown Ge contents (slightly lower than the nominal ones) were obtained using $z$-contrast HAADF, calibrated with energy-dispersive $\mathrm{x}$-ray analysis of the thick $\mathrm{SiGe}$ buffer layer. The parameters thus obtained were used to calculate the hole subband structure using our fully anisotropic $6 \times 6 \mathbf{k} \cdot \mathbf{p}$ method, ${ }^{14}$ which in both cases yielded an interwell transition energy of $\sim 20 \mathrm{meV}$ between the two lowermost $\mathrm{HH}$ states in the two wells, in good agreement with the measured wavelengths for the maximum pumpprobe signal $(62.5 \mu \mathrm{m}, \sim 19.8 \mathrm{meV}$ for BF1499 and $60.1 \mu \mathrm{m}, \sim 20.6 \mathrm{meV}$ for BF1500). The calculated subband structure of the as-grown CAQWs in sample BF1499 is presented in Fig. 2, where the diagonal nonradiative transition across the $i$-Si barrier layer is indicated by a red arrow. The slightly different depths of the two wells shown in Fig. 2 reproduce the slight difference in Ge content between them found in our HAADF measurements in all periods imaged. The lifetimes were measured using the Dutch Free Electron Laser for Infrared eXperiments (FELIX), in the Netherlands. The FELIX delivers macropulses with a length of $7 \mu \mathrm{s}$ at a repetition rate of $5 \mathrm{~Hz}$. Each macropulse consists of a train of micropulses of duration $\sim 10 \mathrm{ps}$ and $25 \mathrm{MHz}$ repetition rate. A pump-probe technique (or transient bleaching technique) in which the FELIX beam is split into pump, probe, and reference beams was used. ${ }^{15}$ The samples were mounted in vacuum on the cold finger of a liquid helium continuousflow cryostat, with temperature variable from 4.2 to $300 \mathrm{~K}$. In order to access the HH-HH transitions it was necessary to pump and probe at oblique incidence, enabling the polarization selection rule $\mathbf{E}$ parallel to the growth direction. The experiments have been performed with the wavelength of FELIX tuned to the absorption peak of the subband transition at $61.3 \mu \mathrm{m}$. Figure 3 shows the transient bleaching signal for sample BF1499 as a function of time delay between probe and pump measured for different values of the FELIX pump power at $10 \mathrm{~K}$ (a), and for different temperatures (b).

\section{THEORETICAL MODEL}

IFR is characterized by the average height $\Delta$ and lateral size (correlation length) $\Lambda$ of the Gaussian fluctuation of the 


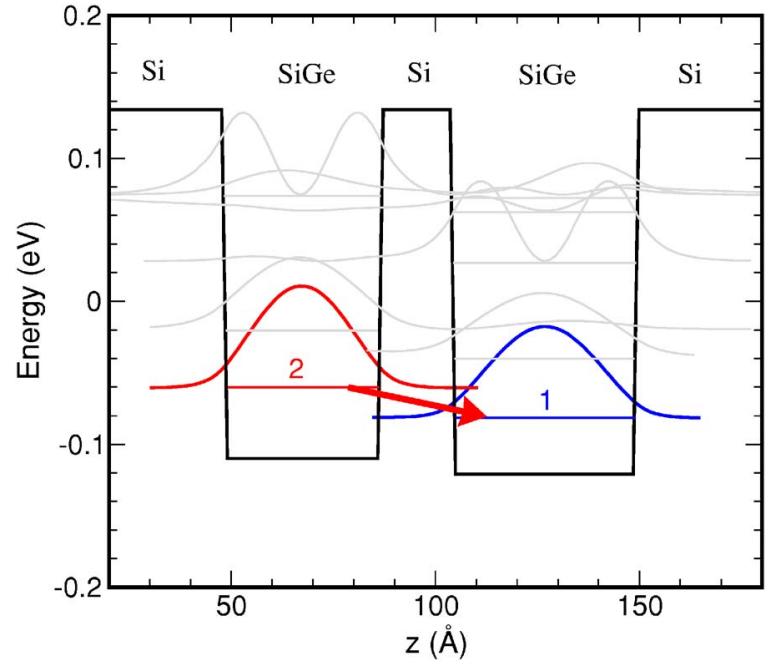

FIG. 2. (Color online) Calculated subband structure of BF1499 showing the energy levels and the square of the wave functions of the first eight hole states (we use an electronlike picture where the energy scale is reversed: the ground state is the lowermost). The slightly different depths of the two wells shown reproduce the slight difference in Ge content between them found in our HAADF measurements in all periods imaged. The interwell nonradiative transition between level 2 (red) and level 1 (blue) is indicated by a red arrow.

local position of the interface with respect to an ideally flat surface, described by the autocorrelation function

$$
S(r)=\left\langle\Delta(\boldsymbol{r}) \Delta\left(\boldsymbol{r}^{\prime}\right)\right\rangle=\Delta^{2} \exp \left(-\frac{\left|\boldsymbol{r}-\boldsymbol{r}^{\prime}\right|^{2}}{\Lambda^{2}}\right) .
$$

A change $\Delta(\boldsymbol{r})$ in the well width $L$ at the position $\boldsymbol{r}=(x, y)$ in the plane causes a fluctuation

$$
\delta E_{0}(\boldsymbol{r})=\frac{\partial E_{0}}{\partial L} \Delta(\boldsymbol{r})
$$

in the carrier quantization energy $E_{0}$. The related scattering matrix element ${ }^{10}$

$$
\left|\left\langle m \boldsymbol{k}^{\prime}\left|\delta E_{0}\right| n \boldsymbol{k}\right\rangle\right|^{2}=\left|\int d^{2} r F_{m n} \Delta(\boldsymbol{r}) e^{i \boldsymbol{q} \cdot \boldsymbol{r}}\right|^{2}
$$

(where $F_{m n}$ contains the product of the wave functions and the potential jump $\delta E_{0}$ at the interface) is proportional to the Fourier transform of the autocorrelation function (1),

$$
S(q)=\pi \Delta^{2} \Lambda^{2} \exp \left(-\frac{q^{2} \Lambda^{2}}{4}\right) .
$$

If the Fermi wave vector is much smaller than $\Lambda^{-1}$ (the effective cutoff wave vector for the momentum exchange $q$ ), then $S(q) \approx \pi(\Delta \Lambda)^{2}$. In this limit the matrix elements depend only on the product $\Delta \Lambda$. This is, however, not the case with our samples and, for a given value of the product $\Delta \Lambda=p_{0} \AA^{2}$, we have found large differences between the lifetimes calculated with $\left[\Delta=1 \AA, \Lambda=p_{0} \AA\right]$ and $\left[\Delta=4 \AA, \Lambda=p_{0} / 4 \AA\right]$, which increased with decreasing carrier temperature.
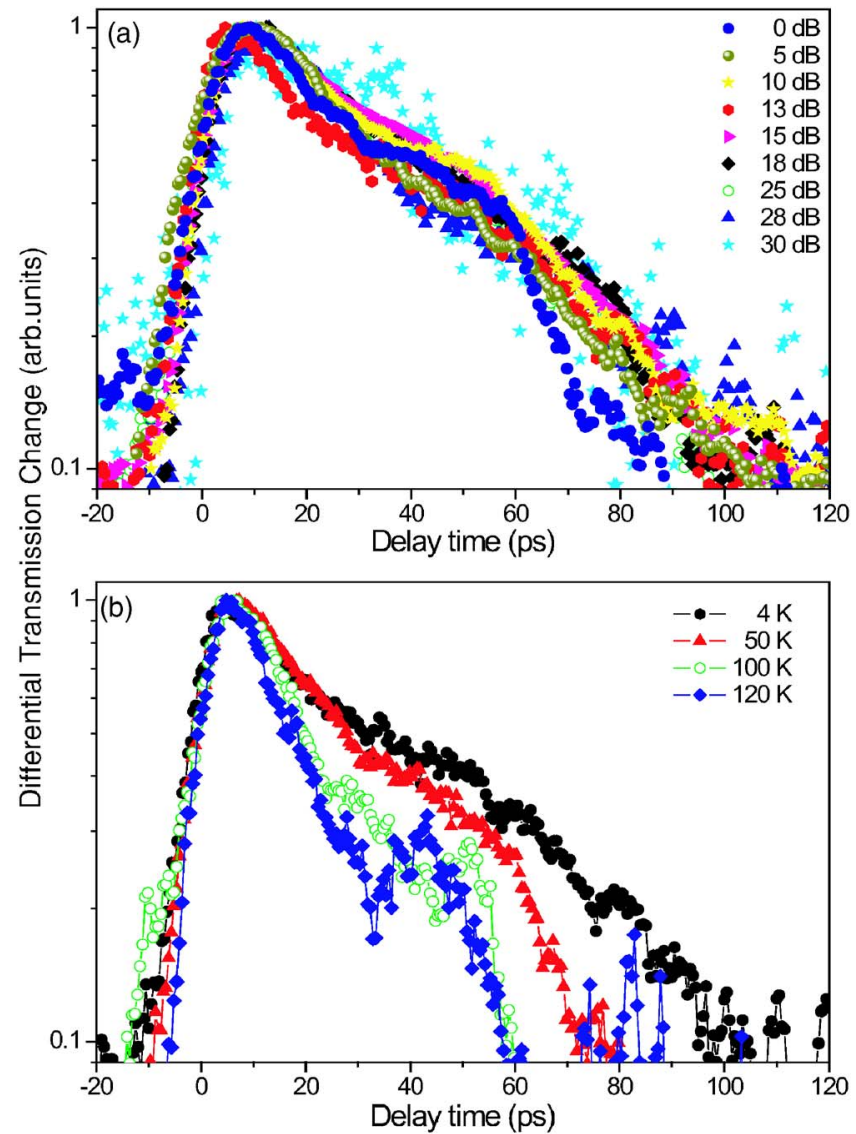

FIG. 3. (Color online) Power (a) and temperature (b) dependence of the interwell transition lifetimes, measured at $T=4 \mathrm{~K}$ for BF1499. In (a) the power decreases with attenuation from 0 to $30 \mathrm{~dB}$.

Calculation of the scattering rates is more involved in the valence than in the conduction band, due to the complicated nature of the band structure. The expression for the IFR scattering matrix element Eq. (2) used here, for example, is similar to that used by Unuma et al. for the case of electrons; ${ }^{10}$ however, in the present work the quantity $F_{m n}$ had to be modified to allow each of the wave function components to experience a different potential at the interface, according to its (heavy-hole, light-hole, or split-off) character. The transition rates due to optical and acoustic phonon, alloy disorder, and hole-hole scattering were calculated as described previously. ${ }^{14,16}$ The total scattering rates due to all the decay mechanisms were then employed in a multisubband rate equation model ${ }^{14,16}$ to track the time evolution of hole population over subbands, following the initial excitation.

\section{RESULTS AND DISCUSSION}

Due to the lack of detailed experimental information on the quality of our samples interfaces, we resorted to the standard ${ }^{8,12,17}$ procedure of treating $\Delta$ and $\Lambda$ as fitting parameters. We deduced their values from the temperature dependence of the decay time in one structure (BF1499) and then used them in the calculation of the lifetimes of the other structure (BF1500), obtaining very good agreement with our 


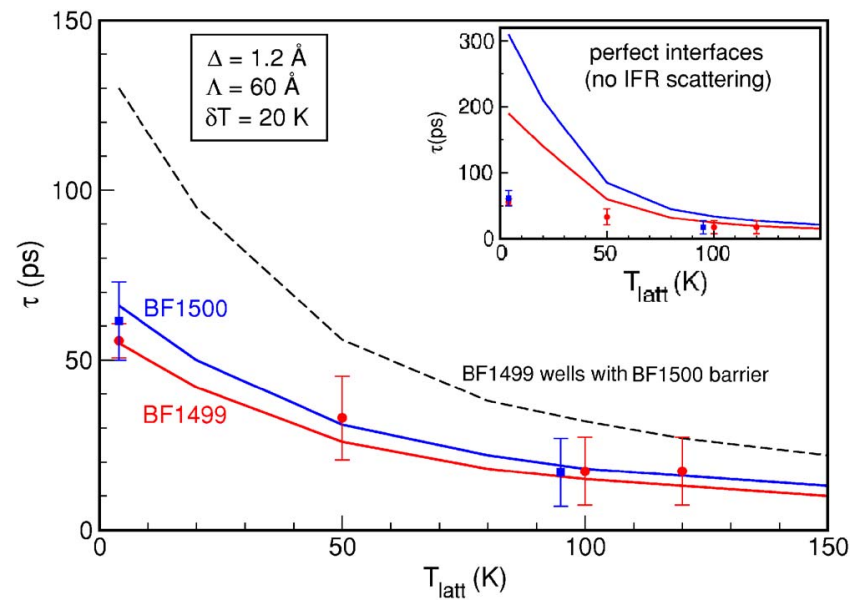

FIG. 4. (Color online) Comparison of experimentally measured (symbols) and theoretically predicted (lines) interwell transition lifetimes as a function of lattice temperature $T_{\text {latt }}$ for our two samples BF1499 (red) and BF1500 (blue). The red symbols are extracted from single exponential fits to the data in Fig. 3(b). The dashed line represents the calculated lifetime for a hypothetical structure with the wells of BF1499 and the barrier of BF1500. Inset: lifetimes calculated for BF1500 and BF1499 in the case of perfect interfaces, i.e., in the absence of IFR scattering (the experimental data are the same as in the main frame). All curves were obtained using $\Delta=1.2 \AA, \Lambda=60 \AA$, and $T_{h}=T_{\text {latt }}+20 \mathrm{~K}$.

measured data, as shown in Fig. 4. Our best fits are obtained with $\Delta=1.2-1.5 \AA$ and $\Lambda=40-60 \AA$ suggesting goodquality interfaces. These IFR parameters are also within the range of values reported in the literature ${ }^{9,11,12,18}(\Delta=1-4 \AA$, $\Lambda=23-300 \AA, \Delta \Lambda=60-1200 \AA^{2}$ ) for $\mathrm{Si} / \mathrm{SiGe}$ interfaces with various Ge content. Interestingly, the value $\Delta=1.5 \AA$ was also assumed by Leuliet et al. in their study of GaAs/ $\mathrm{Al}_{x} \mathrm{Ga}_{1-x} \mathrm{As} \mathrm{QCLs},{ }^{13}$ to obtain the best agreement between theoretical and experimental total scattering rates. In all our calculations we also assume the carrier temperature $T_{h}$ at detection time to be related to the lattice temperature $T_{\text {latt }}$ according to $T_{h}=T_{\text {latt }}+\delta T$, where $\delta T$ is a constant (i.e., $T_{\text {latt }}$-independent) quantity representing the residual effects of the carrier heating produced by the laser pulse. We used $\delta T$ as an additional fitting parameter and found that the choice $\delta T=0-20 \mathrm{~K}$ gives the best fit to experiment. This assumption is not unreasonable, as the hole intrasubband thermalization occurs on a subpicosecond time scale whereas the experimental time resolution is in the picosecond range, allowing access to carriers already relaxed to (or near) the bottom of the subband. A further confirmation of this fast dynamics is provided by the almost power-independent behavior shown by our measured subband lifetimes over a range of attenuation of $30 \mathrm{~dB}$ [see Fig. 3(a)], indicating a very fast intrasubband carrier thermalization time, experimentally inaccessible by our present setup. In contrast the same lifetimes display a clear dependence on the lattice temperature [see Fig. 3(b)] confirming that we are probing thermalized carriers. However our samples exhibit a slower variation with temperature compared to equivalent GaAsbased systems, ${ }^{2}$ where polar optical phonon scattering dominates.
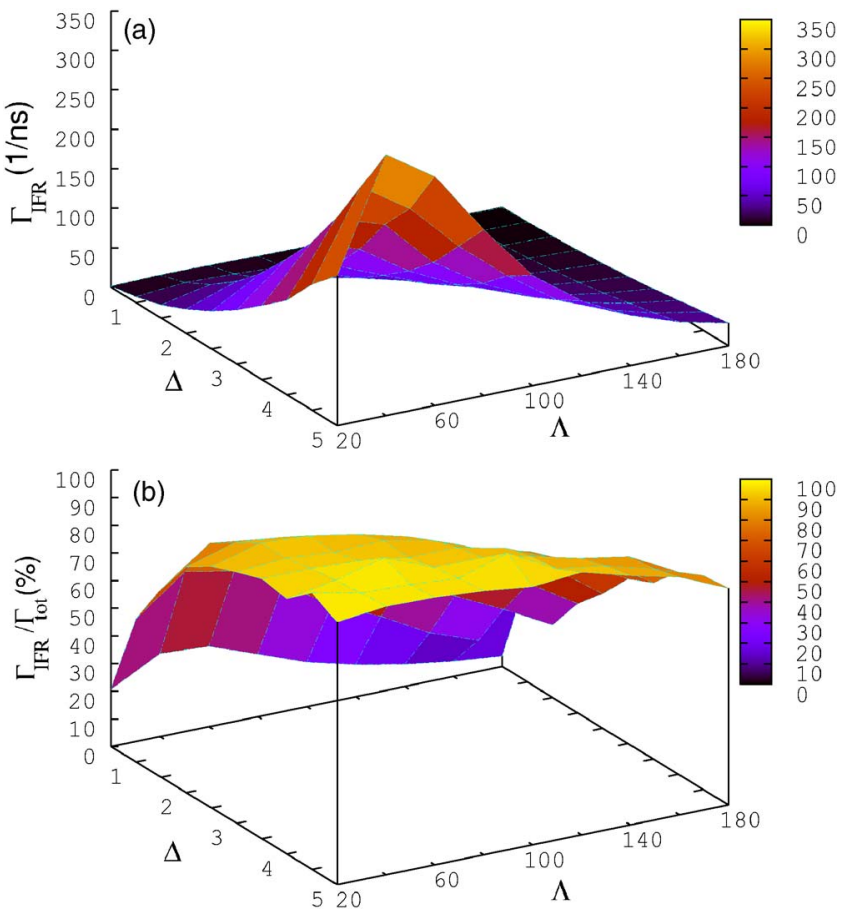

FIG. 5. (Color online) Absolute (a) and relative (b) IFR scattering rates as a function of the IFR parameters $\Delta$ and $\Lambda$ calculated for BF1499 at $T_{\text {latt }}=20 \mathrm{~K}$, assuming a carrier temperature of $40 \mathrm{~K}$.

Figure 5 shows both absolute (a) and relative (b) IFR scattering rates as a function of $\Delta$ and $\Lambda$ calculated for BF1499 at a lattice temperature of $20 \mathrm{~K}$ (similar results are obtained for BF1500). For a fixed value of $\Delta$, the IFR rates increase initially as $\Lambda^{2}$, reach a maximum around $\Lambda=50 \AA$, and then decrease as $\exp \left(-q^{2} \Lambda^{2} / 4\right)$. The dependence on $\Delta$ is instead quadratic for all values [see Eq. (3)]. The total scattering rates have a similar magnitude, indicating that IFR is the dominant scattering mechanism in these structures at low temperature. This is confirmed by the results presented in Fig. 6, showing the [absolute (a), and percentile (b)] contributions to the total scattering rates coming from the different scattering mechanisms, acoustic and optical (op1=Ge-Ge, op2 $=\mathrm{Si}-\mathrm{Si}$, and op3 $=\mathrm{Si}-\mathrm{Ge}$ branches) phonons, alloy disorder (i.e., composition fluctuations), IFR, and hole-hole interaction, calculated as a function of the lattice temperature $T_{\text {latt }}$ for $\Delta=1.2 \AA, \Lambda=60 \AA$, and $\delta T=20 \mathrm{~K}$. In the absence of IFR, the main scattering mechanisms at low temperature are (in order of decreasing importance): carrier-carrier, alloy disorder, acoustic phonons, and optical phonons (Ge-Ge, Si-Ge, and $\mathrm{Si}-\mathrm{Si}$ respectively). While carrier-carrier and phonon scattering increase with temperature as expected (the latter much faster than the former), the scattering rate due to alloy disorder shows a slight temperature dependence owing to the temperature dependence of the hole distribution.

From Fig. 6(a) we see that when $T_{\text {latt }}$ increases other mechanisms, namely, acoustic and optical phonon (first the Ge-Ge and then the Si-Ge branches) and hole-hole scattering, become important. For $T_{\text {latt }}>100-150 \mathrm{~K}$ they are comparable in magnitude to IFR, and for $T_{\text {latt }}>230 \mathrm{~K}$ both acoustic and Ge-Ge optical phonon scattering rates become larger than IFR rates. However from Fig. 6(b) we see that 


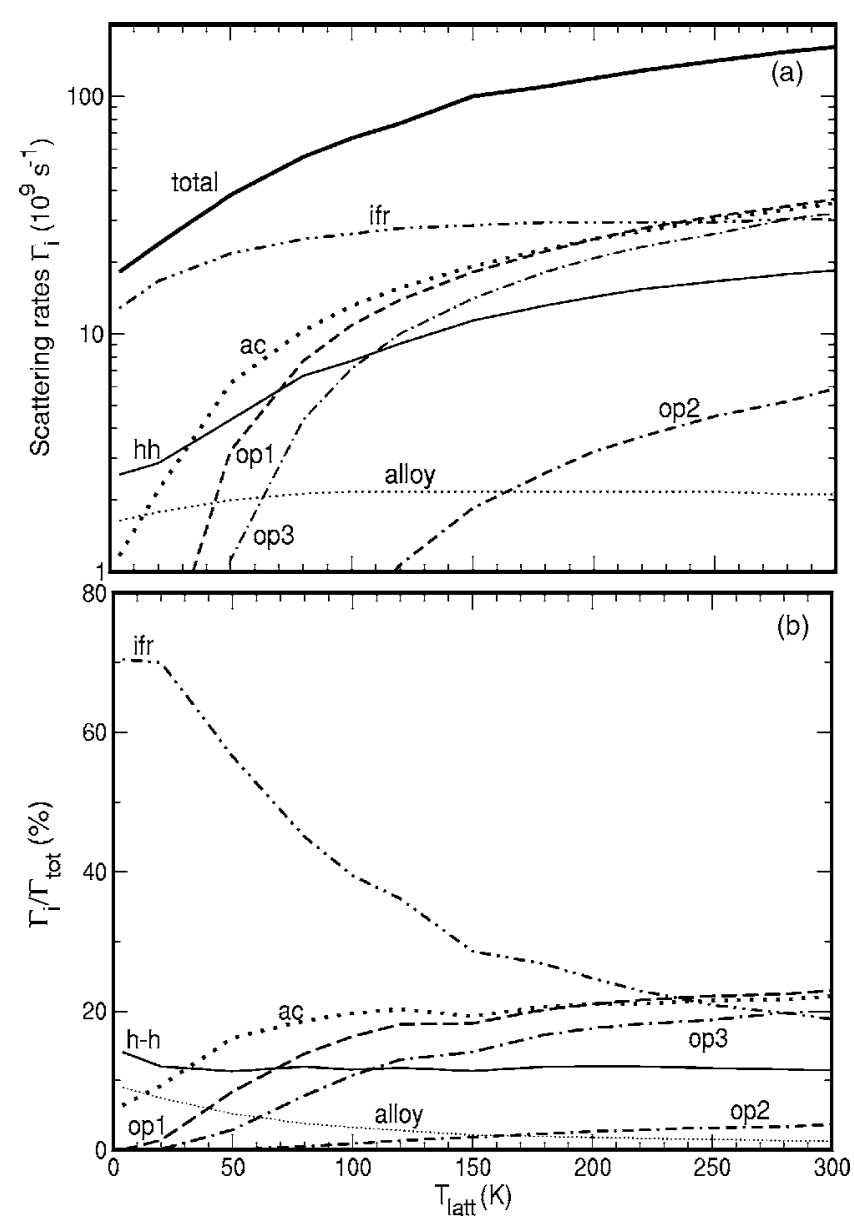

FIG. 6. Total scattering rate calculated for BF1499 as a function of the lattice temperature $T_{\text {latt }}$ for $\Delta=1.2 \AA, \Lambda=60 \AA$, and $\delta T$ $=20 \mathrm{~K}$, decomposed into absolute (a) and percentile (b) contributions from the different scattering mechanisms: acoustic (ac) and optical (op1 $=\mathrm{Ge}-\mathrm{Ge}$, op $2=\mathrm{Si}-\mathrm{Si}$, and op $3=\mathrm{Si}-\mathrm{Ge}$ branches) phonons, alloy disorder, IFR, and hole-hole interaction.

already for $T_{\text {latt }}>70 \mathrm{~K}$ the IFR contribution becomes less than $50 \%$ of the total scattering rate, indicating that, although still comparatively large, IFR is no longer the dominant factor influencing the subband carrier lifetimes in this temperature range. As shown in Fig. 7, at room temperature, the contribution of IFR to the total scattering rate reaches $50 \%$ for $\Delta=2.5$, but it is only for $\Delta>3.5$ that such contribution is above $50 \%$ for all values of $20 \leqslant \Lambda \leqslant 180$. IFR rates are, however, already larger than those of any other mechanism for $\Delta \geqslant 1.75$. In contrast, we find that at low temperature $(=20 \mathrm{~K})$ it is only when $\Delta$ drops below $0.6 \AA$ (i.e., for a very good quality interface) that hole-hole, alloy, and acoustic phonon scattering become stronger, for most values of $\Lambda$.

Having proved the importance of IFR at low temperature, we now need to go back to Fig. 4 and discuss an apparent inconsistency. Although the CAQWs in BF1499 and BF1500 have different layer thicknesses, we see that their measured lifetimes are the same within experimental error. This is counterintuitive as, in a regime where scattering is dominated by IFR, one would expect the transition rates to increase with decreasing barrier width, as IFR rates are proportional to the value of the wave functions at the interface
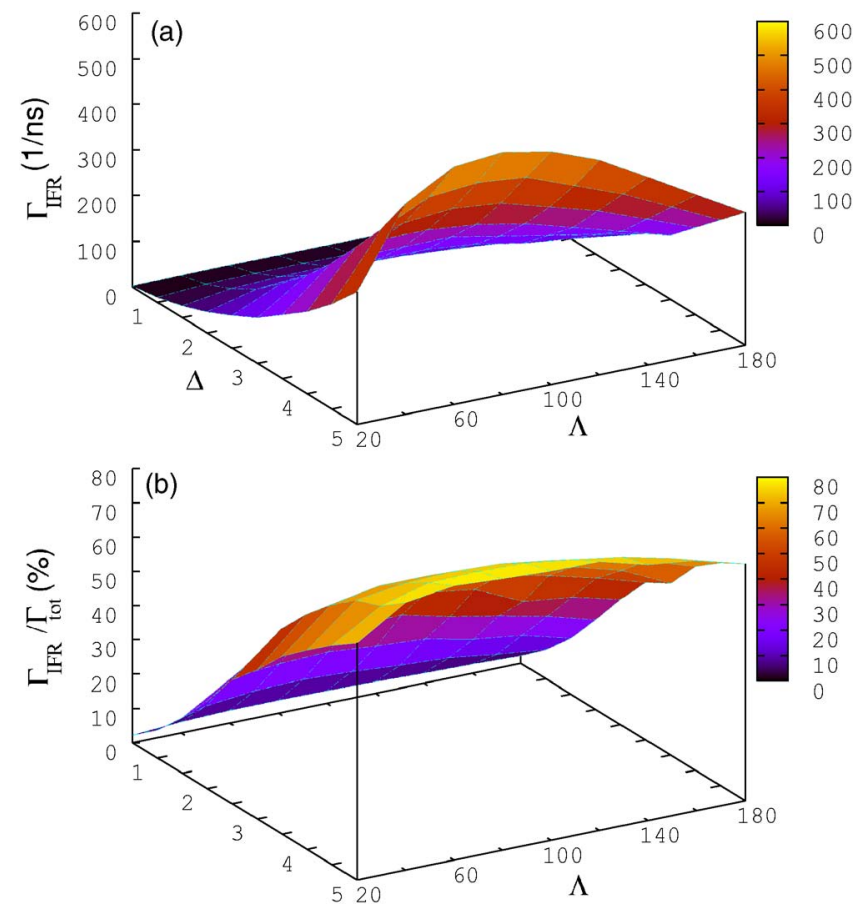

FIG. 7. (Color online) Absolute (a) and relative (b) IFR scattering rates as a function of the IFR parameters $\Delta$ and $\Lambda$ calculated for $\mathrm{BF} 1499$ at $T_{\text {latt }}=300 \mathrm{~K}$, assuming a carrier temperature of $320 \mathrm{~K}$.

(closely related to their penetration into the barrier) which is larger for thinner barriers. The observed behavior can, however, be explained by noting that the structure with the thinnest barrier (BF1499) also has the widest wells. The confinement of the wave functions is therefore reduced in BF1499 compared to BF1500, which would result in a reduced value at the interface if the barrier dimensions were the same. In this case the lifetime would be longer, as shown by the dashed line in Fig. 4, obtained for a hypothetical structure with the wells of BF1499 and the barrier of BF1500. In the case of BF1499, however, the narrower barrier allows a larger penetration and the value of the wave function at the interface becomes similar in both samples. This effect is well reflected by our calculated lifetimes, showing that both barriers and wells need to be carefully engineered in order to tailor the intersubband lifetimes to the desired values. The effect on the lifetime of increasing the barrier width is obtained from a comparison of the dashed and the red lines in Fig. 4, whereas comparing the dashed and the blue lines shows the effect of widening the wells. We see that an increase of $0.5 \mathrm{~nm}$ in the barrier width is more effective in reducing the transition rate than the same increase in the width of one of the wells (and a $0.2 \mathrm{~nm}$ increase in the other).

Further evidence that the inclusion of IFR scattering is crucial to explain the intersubband decay dynamics in SiGe heterostructures is provided by the results presented in the inset of Fig. 4, where the lifetimes for BF1500 and BF1499 calculated for perfect interfaces (i.e., in the absence of IFR) are plotted as a function of lattice temperature and compared with our measured data (the same as in the main panel). We see that both (i) the absolute values of the lifetimes of the 
two samples and (ii) their ratio are in plain disagreement with experiment. Point (ii) here is the most meaningful, as it clearly shows that, apart from its evident quantitative disagreement with our measurements, the observed behavior of the lifetimes as a function of temperature cannot even qualitatively be described without taking into account the effects of IFR scattering. This feature excludes any possibility of attributing (i) to inaccuracies in our scattering calculations and suggests that the dominant role of IFR scattering in intersubband transitions, shown here for energies below the optical phonon energy in multiple quantum well systems and already found in mid-infrared $\mathrm{GaAs} / \mathrm{Al}_{x} \mathrm{Ga}_{1-x} \mathrm{As} \mathrm{QCLs},{ }^{13}$ is a general property of a wide range of semiconductor heterostructures ranging from III-V to IV-IV materials.

\section{CONCLUSIONS}

In summary, we presented a theoretical and experimental study of the nonradiative lifetime of interwell transitions between heavy-hole subbands spaced by less than the (Ge-Ge) optical phonon energy in two different $p$-SiGe coupled asymmetric quantum wells. The observed lifetimes were found to depend on the lattice temperature, but showed no dependence on the excitation power over a wide range of pump intensities, indicating a subpicosecond intrasubband cooling time, well below our experimental resolution. However, the reduction of the lifetimes with increasing temperature was significantly smaller than that observed in GaAs devices $^{2}$ due to the lack of polar optical phonon scattering.
Our calculated transition rates included the contributions from phonon, alloy disorder, IFR, and hole-hole scattering. An average step height $\Delta=1.2-1.5 \AA$ and a correlation length $\Lambda=40-60 \AA$ were deduced by comparison with experiment, implying that our interfaces were of good quality. In spite of that we found IFR to be the strongest scattering mechanism up to about $200 \mathrm{~K}$. In this range, variations as small as 1 monolayer in the average dimensions of both barrier and wells were predicted to impact significantly on the interwell transition rates, in very good agreement with the measured data. At low temperature, IFR scattering rates were found to be larger than those of any other process for as small a step as $0.6 \AA$. By comparing our results with those obtained in a recent study ${ }^{13}$ we concluded that, for the interface quality currently achievable experimentally in III-V and IV-IV heterostructures, IFR will dominate all other scattering mechanisms for transitions below the optical phonon energy at low temperatures.

\section{ACKNOWLEDGMENTS}

This work has been supported by grants from EPSRC (Grant No. GR/S27528), DTI (DTI-MNT contract No. 491 "Fast THz Cameras Using Micro- and Nano-Technology"), and the EU project SHINE (IST-2001-38035). We also gratefully acknowledge support from EPSRC for the U.K. program at FELIX and from the Stichting voor Fundamenteel Onderzoek der Materie (FOM) for providing the required beam time on FELIX. The skillful assistance by the FELIX staff was highly appreciated.
${ }^{1}$ F. Capasso et al., IEEE J. Quantum Electron. 38, 511 (2002).

${ }^{2}$ B. S. Williams, S. Kumar, H. Callebaut, Q. Hu, and J. L. Reno, Appl. Phys. Lett. 83, 5142 (2003).

${ }^{3}$ L. Ajili et al., Appl. Phys. Lett. 85, 3986 (2004).

${ }^{4}$ R. W. Kelsall, Z. Ikonic, P. Murzyn, C. R. Pidgeon, P. J. Phillips, D. Carder, P. Harrison, S. A. Lynch, P. Townsend, D. J. Paul, S. L. Liew, D. J. Norris, and A. G. Cullis, Phys. Rev. B 71, 115326 (2005); P. Murzyn, C. R. Pidgeon, J.-P. R. Wells, I. V. Bradley, Z. Ikonic, R. W. Kelsall, P. Harrison, S. A. Lynch, D. J. Paul, D. D. Arnone, D. J. Robbins, D. Norris, and A. G. Cullis, Appl. Phys. Lett. 80, 1456 (2002).

${ }^{5}$ S. A. Lynch, R. Bates, D. J. Paul, D. J. Norris, A. G. Cullis, Z. Ikonic, R. W. Kelsall, P. Harrison, D. D. Arnone, and C. R. Pidgeon, Appl. Phys. Lett. 81, 1543 (2002); R. Bates, S. A. Lynch, D. J. Paul, Z. Ikonic, R. W. Kelsall, P. Harrison, S. L. Liew, D. J. Norris, A. G. Cullis, W. R. Tribe, and D. D. Arnone, ibid. 83, 4092 (2003).

${ }^{6}$ I. Bormann, K. Brunner, S. Hackenbuchner, G. Abstreiter, S. Schmult, and W. Wegscheider, Appl. Phys. Lett. 83, 5371 (2003).

${ }^{7}$ R. E. Prange and T. W. Nee, Phys. Rev. 158, 779 (1968).
${ }^{8}$ H. Sakaki, T. Noda, K. Hirakawa, M. Tanaka, and T. Matsusue, Appl. Phys. Lett. 51, 1934 (1987).

${ }^{9}$ U. Penner, H. Rucker, and I. N. Yassievich, Semicond. Sci. Technol. 13, 709 (1998).

${ }^{10}$ T. Unuma, M. Yoshita, T. Noda, H. Sakaki, and H. Akiyama, J. Appl. Phys. 93, 1586 (2003).

${ }^{11}$ S. Tsujino et al., Appl. Phys. Lett. 84, 2829 (2004).

${ }^{12}$ S. Tsujino et al., Appl. Phys. Lett. 86, 062113 (2005).

${ }^{13}$ A. Leuliet, A. Vasanelli, A. Wade, G. Fedorov, D. Smirnov, G. Bastard, and C. Sirtori, Phys. Rev. B 73, 085311 (2006).

${ }^{14}$ Z. Ikonić, P. Harrison, and R. W. Kelsall, Phys. Rev. B 64, 245311 (2001).

${ }^{15}$ P. C. Findlay, C. R. Pidgeon, R. Kotitschke, A. Hollingworth, B. N. Murdin, C. J. G. M. Langerak, A. F. G. van der Meer, C. M. Ciesla, J. Oswald, A. Homer, G. Springholz, and G. Bauer, Phys. Rev. B 58, 12908 (1998).

${ }^{16}$ Z. Ikonić, P. Harrison, and R. W. Kelsall, J. Appl. Phys. 96, 6803 (2004).

${ }^{17}$ T. Ando, A. B. Fowler, and F. Stern, Rev. Mod. Phys. 54, 437 (1982).

${ }^{18}$ R. M. Feenstra et al., J. Vac. Sci. Technol. B 13, 1608 (1995). 